\title{
Malformação de Chiari e a sua correlação com transtornos orofaciais
}

\author{
Chiari malformation and its correlation with orofacial disorders \\ Malformación de Chiari y su correlación con trastornos orofaciales
}

\section{Resumo}

Introdução: A malformação de Chiari trata-se da herniação do tecido cerebelar através do forame magno, podendo levar a uma série de sinais e sintomas que podem afetar qualitativamente a vida do portador dessa síndrome. Por apresentar muitos sintomas, estes podem direcionar ao diagnóstico e tratamento incorreto se não houver o conhecimento adequado. Objetivo: este estudo tem o objetivo de correlacionar os achados clínicos orofaciais em pacientes que apresentam a malformação de Chiari e seu diagnóstico diferencial com DTM. Metodologia: Realizou-se uma revisão literária através do cruzamento dos descritores selecionados em busca eletrônica, encontrados nos periódicos das bases de dados SCIELO, LILACS, SCOPUS e MEDLINE. Discussão: A Malformação de Chiari apresenta um grupo de malformações congênitas que afetam diretamente a qualidade de vida dos pacientes. Esta condição pode estar associada à neuralgia trigeminal, porém seu mecanismo exato ainda não é claramente compreendido. A ressonância magnética é o exame de imagem padrão ouro para o diagnóstico desta condição e, a escolha pela terapia ideal, tanto o manejo clínico quanto o cirúrgico, deve ser corretamente indicado em pacientes sintomáticos, pela chance, embora rara, da possível diminuição espontânea ou resolução completa da herniação tonsilar. Conclusão: As manifestações clínicas da Malformação de Chiari são variáveis, podendo apresentar semelhanças e até mesmo serem confundidas com outras condições do sistema estomatognático como a DTM. Dessa forma, é importante o conhecimento acerca das suas manifestações clínicas, para que possa ser corretamente diagnosticada e melhor tratada.

Palavras-chave: Malformação de Arnold-Chiari; Neuralgia do trigêmeo; Síndrome da articulação temporomandibular. 


\begin{abstract}
Introduction: Chiari malformation is the herniation of the cerebellar tissue through the foramen magnum, which can lead to a series of signs and symptoms that can qualitatively affect the life of the person with this syndrome. Because they have many symptoms, they can lead to incorrect diagnosis and treatment if there is not adequate knowledge. Objective: this study aims to correlate the orofacial clinical findings in patients with Chiari malformation and its differential diagnosis with TMD. Methodology: A literary review was carried out by crossing the selected descriptors in electronic search, found in the journals of the databases SCIELO, LILACS, SCOPUS and MEDLINE. Discussion: Chiari Malformation presents a group of congenital malformations that directly affect patients' quality of life. This condition may be associated with trigeminal neuralgia, but its exact mechanism is still not clearly understood. Magnetic resonance imaging is the gold standard imaging test for the diagnosis of this condition and the choice for the ideal therapy, both clinical and surgical management, should be correctly indicated in symptomatic patients, due to the chance, although rare, of possible spontaneous or complete resolution of tonsillar herniation. Conclusion: The clinical manifestations of Chiari Malformation are variable, and may present similarities and even be confused with other conditions of the stomatognathic system such as TMD. Thus, knowledge about its clinical manifestations is important, so that it can be correctly diagnosed and better treated.
\end{abstract}

Keywords: Arnold-Chiari Malformation; Trigeminal neuralgia; Temporomandibular joint dysfunction syndrome.

\title{
Resumen
}

Introducción: La malformación de Chiari es la herniación del tejido cerebeloso a través del foramen magnum, lo que puede dar lugar a una serie de signos y síntomas que pueden afectar cualitativamente la vida del paciente. Debido a que tienen muchos síntomas, pueden conducir a un diagnóstico y tratamiento incorrectos si no se cuenta con los conocimientos adecuados. Objetivo: este estudio tiene como objetivo correlacionar los hallazgos clínicos orofaciales en pacientes con malformación de Chiari y su diagnóstico diferencial con TTM. Metodología: Se realizó una revisión literaria cruzando los descriptores seleccionados en búsqueda electrónica, encontrados en las revistas de las bases de datos SCIELO, LILACS, SCOPUS y MEDLINE. Discusión: La Malformación de Chiari presenta un grupo de malformaciones congénitas que afectan directamente la calidad de vida de los pacientes. Esta afección puede estar asociada con la neuralgia del trigémino, pero su mecanismo exacto aún no se comprende con claridad. La resonancia magnética es la prueba de imagen de oro estándar para el diagnóstico de esta afección y la elección de la terapia ideal, tanto el manejo clínico como quirúrgico, debe estar correctamente indicada en pacientes sintomáticos, debido a la posibilidad, aunque rara, de posible aparición espontánea o completa resolución de la hernia amigdalina. Conclusión: Las manifestaciones clínicas de la Malformación de Chiari son variables, pudiendo presentar similitudes e incluso confundirse con otras afecciones del sistema estomatognático como el TTM. Por ello, el conocimiento de sus manifestaciones clínicas es importante, para que pueda ser correctamente diagnosticado y mejor tratado.

Palabras clave: Malformación de Arnold-Chiari; Neuralgia del trigémino; Síndrome de la disfunción de articulación temporomandibular.

\section{Introdução}

Relatos de herniação tonsilar cerebelar e siringomielia (cavidade cística longitudinal na medula espinhal, que pode ocorrer como uma complicação de tumor, meningite, aracnoidite ou trauma), são datados desde o século XIX. Porém, a malformação de Arnold Chiari foi descrita pela primeira vez em espécimes de autópsia pediátrica em 1891, pelo patologista austríaco Hans Chiari (1851-1916) o qual descreveu e classificou de forma inicial a malformação em três tipos: I, II, III e posteriormente o tipo IV. Legado com o nome de seu professor Dr. Arnold, e seu nome, o distúrbio da região posterior do cérebro é denominado como malformação de Arnold Chiari (Cedeño, 2018; Zhao et al., 2016; Souza, 2011; Oliveira, 2011; Ganesh et al., 2014).

Muito tem sido escrito e conjecturado sobre a causa do deslocamento cerebelar nesta condição, mas até o momento, nenhuma causa definitiva única foi identificada (Mcclugage, 2019; Ganesh et al., 2014). Existem inúmeras teorias que levantam a hipótese da etiologia dessa síndrome, tanto de causas fisiológicas primárias, bem como síndromes adquiridas secundárias que levam a mudanças fisiológicas (Mcvige; Leonardo, 2015). Porém, tradicionalmente, esta condição é descrita como uma malformação, o que pode indicar uma relação causal na forma de um defeito genético ou anormalidade do desenvolvimento. No entanto, há poucas evidências de que seja uma malformação congênita, pois raramente é detectado em recém-nascidos ou bebês, e é incomum os portadores serem da mesma família (Fric; Ringstad; Eide, 2019). 
Como citado anteriormente, esta síndrome pode ser classificada em 4 grupos. A do tipo I consiste na herniação do tecido cerebelar através do forame magno por pelo menos 3-5 mm. A tipo II é caracterizada pela inferiorização da medula, do quarto ventrículo e do cerebelo para o canal cervical espinhal, além de apresentar um crescimento da ponte e do quarto ventrículo (Araújo, 2017; Duarte, 2018). A tipo III consiste na herniação do tronco encefálico e do cerebelo sobre uma meningocele cervical alta (Araújo, 2017). Já a malformação tipo IV, está relacionada ao reduzido desenvolvimento cerebelar, com ausência de herniação, podendo estar associada à siringomielia ou siringo-hidromielia (SH) em 30 a $70 \%$ dos casos (Mcvige; Leonardo, 2015; Araújo, 2017). Mesmo raros, os tipos II, III e IV geralmente são identificados antes ou ao nascimento e podem ser letais (Oliveira, 2011)

De acordo com a classificação, apesar de raro, o tipo mais comum é a malformação de Chiari tipo I (MCI) (Mcvige; Leonardo, 2015), que pode ser encontrada em aproximadamente $0,9 \%$ da população adulta e $0,6 \%$ da população pediátrica (Mcclugage, 2019). Ainda, apresenta maior incidência em pacientes do sexo feminino, principalmente em pacientes jovens, podendo vir a se tornar sintomática, na maioria dos casos, entre a quarta e a sexta década de vida (Oliveira, 2011).

O tratamento da malformação de Chiari pode ser cirúrgico ou conservador, a depender da presença ou ausência de sintomatologia, dos achados neurológicos, do nível de ectopia tonsilar e se houver, ou não, comprometimento do estilo de vida (Poretti, 2016, Langridge, 2017, Briganti, 2013). Porém, esse tratamento só pode ser definido após o correto diagnóstico desta síndrome, que pode ser dificultado pelo fato da apresentação da MCI ser semelhante a outras condições que acometem a face, como a Disfunção Temporomandibular (DTM). Sendo assim, este estudo tem o objetivo de correlacionar os achados clínicos orofaciais em pacientes que apresentam a malformação de Chiari e seu diagnóstico diferencial com DTM.

\section{Metodologia}

O presente estudo trata-se de uma revisão da literatura, desenvolvida por meio de fontes indexadas nas bases de dados do Scientific Eletronic Library Online (SCIELO), Literatura Latino Americana e do Caribe em Ciências da Saúde (LILACS) e Medical Literature Analysis and Retrival System Online (MEDLINE) e Google Scholar. Buscou-se por estudos publicados entre o período de 2011 e 2021, fazendo um cruzamento entre os descritores "Malformação de Arnold-Chiari", "Neuralgia do Trigêmeo" e "Síndrome da Articulação Temporomandibular". Os artigos foram pesquisados nos idiomas português, inglês e espanhol e a partir de sua análise na íntegra, foram selecionados e contribuíram para a análise descritiva deste trabalho.

\section{Resultados e Discussão}

A malformação de Chiari é considerada uma síndrome por apresentar um grupo de malformações congênitas, envolvendo estruturas que levam a uma série de sinais e sintomas significativos para a qualidade de vida do paciente (ZHAO et al., 2016). A literatura afirma que estes sinais e sintomas clínicos ocorrem quando cicatrizes e aderências aracnóides se acumulam no forame magno, possivelmente devido ao atrito das tonsilas cerebelares contra o osso ao longo dos anos e pela compressão do cérebro posterior por essas aderências (Mcvige; Leonardo, 2015). O deslocamento tonsilar cerebelar também pode aumentar quando associado à presença de processos patológicos intracranianos, como hidrocefalia, malformação arteriovenosa, lesão de massa ou hipotensão (Mcvige; Leonardo, 2015).

Dentre os sinais e sintomas pode-se encontrar: cefaleia, cervicalgia, dor em face, atrofia das papilas linguais, hipestesia facial, neuralgia do trigêmeo ou glossofaríngea, nistagmo, rouquidão, diplopia, zumbido, fraqueza do palato mole, paralisia das cordas vocais. Também, incontinência urinária, parestesia em membros inferiores, fraqueza e distúrbios do sono. Além disso, os sinais clínicos existentes podem ser a compressão do tronco cerebral (levando à síncope, perda auditiva, bradi ou taquicardia), sinais cerebelares (ataxia, dismetria) e lesão da medula espinhal (reflexos hiperativos, espasticidade, clônus, incontinência) (Vasconcelos et al., 2015; Araújo et al., 2018; Mcvige; Leonardo, 2015; Zhao et al., 2016). 
Estes sintomas, principalmente os relacionados à região orofacial, podem levar a um diagnóstico incorreto. A dor ao longo do trajeto do nervo trigêmeo pode estar associada a alterações na pressão intracraniana, afetando o trato trigêmeo que segue dorso-lateralmente à medula espinhal, interrompendo a transmissão nervosa adequada de fibras que medeiam dor e temperatura e mimetizando, consequentemente, os sinais e sintomas de outros quadros patológicos na face (Araújo et al., 2018; Vasconcelos et al., 2015).

Muito frequente na MCI em pacientes adultos e pediátricos, a cefaleia (occipital posterior ou cervical superior) é descrita como uma dor aguda ou latejante exacerbada por tosse, manobra de Valsalva, alterações posturais ou esforço físico (Mcvige; Leonardo, 2015; Mcclugage, 2019). Este é um sintoma de alta relevância que pode se configurar como uma das queixas mais comuns da população geral (Andrade et al., 2019). Porém, em portadores de MCI, quando este sintoma é associado a sintomas de disfunção da articulação temporomandibular, tais como dores no pescoço, zumbidos, perdas auditivas, fala arrastada, disfagia, e distúrbios do sono, é comum o diagnóstico e tratamento inicialmente ser relacionado à DTM.

Vários estudos têm demonstrado que pacientes com sintomas semelhantes aos observados na DTM apresentam outra comorbidade, explicando porque muitos pacientes submetidos a diversos tratamentos continuaram com dor crônica. Assim, é importante que os clínicos que examinam pacientes com sintomas dolorosos na região orofacial considerem qualquer comorbidade, assim como a MCI (Araújo et al., 2018), pois, diagnósticos incorretos podem levar à não resolução e prolongamento dos sinais e sintomas, afetando a qualidade de vida desses pacientes e principalmente daqueles que possuem dores com altas intensidades.

Ainda, a MCI pode coexistir com distúrbios articulares, podendo os sintomas serem amplificados nesses pacientes. Com base nisso, os pacientes com esse distúrbio às vezes podem ser subdiagnosticados, ressaltando a importância do conhecimento dessa malformação por parte de clínicos, dentistas gerais e cirurgiões bucomaxilofaciais (Araújo et al., 2018).

Embora a condição seja incomum, de forma geral, um diagnóstico diferencial clínico e por imagem deve ser estabelecido. Para compreender melhor a relação entre as alterações fisiopatológicas como a causa dos sintomas é necessária a solicitação de determinados exames (Mcvige; Leonardo, 2015). A MCI é frequentemente diagnosticada após achados imaginológicos e clínicos adequados, sendo a ressonância nuclear magnética o melhor exame de escolha, por permitir uma boa visualização do grau de herniação tonsilar cerebelar. Ainda, o diagnóstico pode ser puramente acidental em exames de imagem realizados após um traumatismo craniano, acidente ou infecção. Lewis et al. revisaram retrospectivamente as ressonâncias magnéticas de 599 pacientes com cefaleia, e 24 (0,04\%) foram incidentalmente diagnosticados com MCI (Mcvige; Leonardo, 2015; Mcclugage, 2019; Langridge, 2017, Arruda et al., 2018).

Nesses exames imaginológicos, dos quais a ressonância magnética é o padrão ouro para o diagnóstico (Mcvige; Leonardo, 2015), é feita a medição das estruturas anatômicas por meio de técnicas de neuroimagem, feita das margens internas do forame magno até a parte inferior das amígdalas e verificando o quanto as tonsilas se projetam abaixo da margem do forame magno. Se elas estiverem acima do forame magno, o diagnóstico é normal. Se estiverem $<5 \mathrm{~mm}$ também é normal, mas o termo ectopia tonsilar benigna pode ser usado. Porém, se estiverem $>5 \mathrm{~mm}$, a malformação Chiari I é diagnosticada (Ganesh et al., 2014). As técnicas avançadas de ressonância magnética, como a imagem por tensor de difusão e os estudos de fluxo do LCR, permitem uma melhor compreensão da complexa fisiopatologia subjacente da MCI (Mcvige; Leonardo, 2015).

Tanto o manejo clínico quanto o cirúrgico podem ser usados para tratar o MCI em pacientes sintomáticos, devendo ser ponderados em razão da possível, embora rara, ocorrência de uma diminuição espontânea ou resolução completa da herniação tonsilar (Briganti, 2013). Após o diagnóstico ser estabelecido, o manejo médico inicial pode ser implementado com o uso de farmacoterapia, fisioterapia e injeções terapêuticas (Mcvige; Leonardo, 2015). Porém, a decisão do tratamento cirúrgico é baseada na gravidade e duração dos sintomas do paciente no momento da apresentação, ou seja, é mais considerado para os pacientes que apresentam sintomas progressivos e debilitantes, como aumento da pressão intracraniana, achados neurológicos 
anormais, alterações na drenagem do líquido cefalorraquidiano e persistentes dores de cabeça, relacionadas a episódios de tosses, que interfiram na qualidade de vida (Arruda et al., 2018; Langridge, 2017; Oliveira, 2011). Este tratamento consiste na descompressão do forame magno e, embora haja debate em torno dos aspectos técnicos da cirurgia, os resultados e os riscos da cirurgia estão bem documentados (Langridge, 2017). O novo Índice de Severidade de Chiari (ISC) é um sistema de classificação pré-operatório e pode ser utilizado para prever, em longo prazo, se o paciente melhora com a cirurgia (Cedeño, 2018), e, consequentemente, a sua qualidade de vida.

\section{Considerações Finais}

Os sintomas clínicos da Malformação de Chiari são diversos. Os profissionais de saúde, especialmente os cirurgiões bucomaxilofaciais, devem estar atentos às manifestações orofaciais dessa patologia, que apesar de ter uma rara ocorrência e desconhecimento, permite a possibilidade dos sinais e sintomas serem confundidos com outras condições na face como a DTM, prolongando os seus sinais e sintomas e afetando a qualidade de vida do paciente. Uma abordagem multidisciplinar, tendo em consideração os atuais aspectos clínicos e de imagem, permite um diagnóstico diferencial com doenças neurológicas e tratamento mais rápido, preciso e eficaz.

\section{Referências}

Alperin, N., Loftus, J. R., Oliu, C. J., Bagci, A. M., Lee, S. H., Ertl-Wagner, B., Sekula, R., Lichtor, T. \& Green, B. A. (2015). Imaging-Based Features of Headaches in Chiari Malformation Type I. Neurosurgery, 77(1), 96-103.

Andrade, A. M. C., Souza, D. M. B., Santos, L. R. S., Torres, L. M. N., Silva, M. A. F. \& Fernandes, D. C. (2019). Cefaleia associada à DTM. Ciências Biológicas e de Saúde UNIT, 5 (3), 85-94.

Araújo, I. A., Amorim, E. F., Santana, L. M. M., Alencar, E. P., Araújo, M. I. A., \& Fernandes, P. E. (2017). Malformação de Arnold-Chiari: Uma Revisão Bibliográfica. Journal of Medicine and Health Promotion, 2(3), 661-669.

Briganti, F., Leone, G., Briganti, G., Orefice, G., Caranci, F., \& Maiuri, F. (2013). Spontaneous resolution of Chiari type I malformation. A case report and literature review. The Neuroradiology Journal, 26, 304-309.

Cedeño, E. W., Mora, B. H., Castañeda, J., Sola, J., Criollo, J., Torres, L., Negrete, G. \& Lucas, G. (2018). Arnold Chiari tipo I y siringomielia en el adulto. Revista científica INSPILIP, 2 (1), 1-9.

de Arruda, J. A., Figueiredo, E., Monteiro, J. L., Barbosa, L. M., Rodrigues, C., \& Vasconcelos, B. (2018). Orofacial clinical features in Arnold Chiari type I malformation: A case series. Journal of Clinical and Experimental Dentistry, 10(4), 378-382.

de Souza, R. M., Zador, Z., \& Frim, D. M. (2011). Chiari malformation type I Related conditions. Neurological Research, 33(3), 278-284. https://doi.org/10.1179/016164111X12962202723922.

Duarte, M. L., Abreu, B. F. B. B., Prado, J. L. M. A., J., \& Silva, M. Q. P. (2018). Dyke-Davidoff-Masson Syndrome and Chiari Type Ii Malformation Síndrome De Dyke-Davidoff-Masson E Malformação De Chiari Tipo Ii. Revista Brasileira de Neurologia, 54(4), 34-37.

Durai, R., Fernandes, C., \& Ng, P. C. H. (2010). Arnold-Chiari malformation. British Journal of Hospital Medicine, 71(3), 173. https://doi.org/10.12968/hmed.2010.71.3.46986.

Felício, A. C., Godeiro, C. D. O., Borges, V., Silva, S. M. D. A., \& Ferraz, H. B. (2007). Hemifacial spasm in a patient with neurofibromatosis and ArnoldChiari malformation: A unique case association. Arquivos de Neuro-Psiquiatria, 65(3 B), 855-857. https://doi.org/10.1590/S0004-282X2007000500026

Frič, R., Ringstad, G., \& Eide, P. K. (2019). Chiari-malformasjon type 1-diagnostikk og behandling. Tidsskrift for Den Norske Laegeforening, 139(10). https://doi.org/10.4045/tidsskr.18.0455.

Ganesh, D., Sagayaraj, B. M., Barua, R. K., Sharma, N., \& Ranga, U. (2014). Arnold chiari malformation with spina bifida: A lost opportunity of folic acid supplementation. Journal of Clinical and Diagnostic Research, 8(12), OD01-OD03. https://doi.org/10.7860/JCDR/2014/11242.5335.

Holanda, M. M. A., Neto, B. F. C., Batista, L. L, Kuo, C.-H., Kitamura, M. A. P., \& Valença, M. M. (2019). Lesión como malformación de Chiari secundaria a quiste aracnoidal de la cisterna cuadrigémina. Revista Chilena de Neurocirugía, 42(2), 141-143. https://doi.org/10.36593/rev.chil.neurocir.v42i2.114

Langridge, B., Phillips, E., \& Choi, D. (2017). Chiari Malformation Type 1: A Systematic Review of Natural History and Conservative Management. World Neurosurgery, 104, 213-219. https://doi.org/10.1016/j.wneu.2017.04.082

McClugage, S. G., \& Jerry Oakes, W. (2019). The Chiari I malformation. Journal of Neurosurgery: Pediatrics, 24(3), 217-226. https://doi.org/10.3171/2019.5.PEDS18382. 
Research, Society and Development, v. 10, n. 5, e41010515151, 2021

(CC BY 4.0) | ISSN 2525-3409 | DOI: http://dx.doi.org/10.33448/rsd-v10i5.15151

McVige, J. W., \& Leonardo, J. (2015). Neuroimaging and the Clinical Manifestations of Chiari Malformation Type I (CMI). Current Pain and Headache Reports, 19(6), 4-9. https://doi.org/10.1007/s11916-015-0491-2.

Oliveira, N. F. C. D., Oliveira, R. C. C. D., \& De Paula, J. M. (2011). Manifestações otoneurológicas tardia da malformação de Chiari I. International Archives of Otorhinolaryngology, 15(3), 382-384. https://doi.org/10.1590/s1809-48722011000300018.

Ortiz, L. P., Armas, A. A., Ramos, E. R., \& Rodríguez, L. L. (2018). Un enfoque clínico e histórico de la Malformación de Chiari Tipo I en adultos. Rev. Medica Electron, 40(1), 144-158.

Poretti, A., Ashmawy, R., Garzon-Muvdi, T., Jallo, G. I., Huisman, T. A. G. M., \& Raybaud, C. (2016). Chiari Type 1 Deformity in Children: Pathogenetic, Clinical, Neuroimaging, and Management Aspects. Neuropediatrics, 47(5), 293-307. https://doi.org/10.1055/s-0036-1584563.

Rydell, R. E. \& Pulec, J. L. (1971). Arnold-Chiari Malformation. Arch Otolaryng, 94,8 - 12.

Than, K. D., Sharifpour, M., Wang, A. C., Thompson, B. G., \& Pandey, A. S. (2011). Chiari I malformation manifesting as bilateral trigeminal neuralgia: Case report and review of the literature. Journal of Neurology, Neurosurgery and Psychiatry, 82(9), 1058-1059. https://doi.org/10.1136/jnnp.2009.196121.

Vasconcelos, B. C. E., Barbosa, L. M., Monteiro, J. L. G. C., Gouveia, M. V. C., Caldas, L. C. B. (2015). Interface entre a síndrome de Chiari e a sintomatologia facial. J Braz Coll Oral Maxillofac Surg, 1(2), 503.

Zagzoog, N., \& Reddy, K. (Kesh). (2019). Use of Minimally Invasive Tubular Retractors for Foramen Magnum Decompression of Chi ari Malformation: A Technical Note and Case Series. World Neurosurgery, 128, 248-253. https://doi.org/10.1016/j.wneu.2019.04.094.

Zhao, J. L., Li, M. H., Wang, C. L., \& Meng, W. (2016). A Systematic Review of Chiari I Malformation: Techniques and Outcomes. World Neurosurgery, 88, 7-14. https://doi.org/10.1016/j.wneu.2015.11.087. 